\title{
Révoltes collégiennes, élites juvéniles et société post-révolutionnaire (1815-1848)
}

School revolts, juvenile elites and post-revolution society (1815-1848)

Aufstände von Gymnasiasten, junge Eliten und postrevolutionäre Gesellschaft

(1815-1848)

Revueltas colegiales, élites juveniles y sociedad pos-revolucionaria (1815-1848)

\section{Jean-Claude Caron}

\section{OpenEdition}

Journals

Édition électronique

URL : https://journals.openedition.org/histoire-education/541

DOI : $10.4000 /$ histoire-education.541

ISSN : 2102-5452

Éditeur

ENS Éditions

Édition imprimée

Date de publication : 1 avril 2008

Pagination : 83-108

ISBN : 978-2-7342-1117-4

ISSN : 0221-6280

\section{Référence électronique}

Jean-Claude Caron, «Révoltes collégiennes, élites juvéniles et société post-révolutionnaire

(1815-1848) », Histoire de l'éducation [En ligne], 118| 2008, mis en ligne le 01 janvier 2013, consulté le

20 mai 2021. URL : http://journals.openedition.org/histoire-education/541 ; DOI : https://doi.org/

10.4000/histoire-education.541 


\title{
Révoltes collégiennes, élites juvéniles et société post-révolutionnaire (1815-1848)
}

\author{
Jean-Claude CARON
}

L'histoire de l'instruction, comme celle de l'éducation, ne peut faire l'économie d'une réflexion sur la violence. Au-delà des regards critiques posés sur l'école comme sur la famille par des historiens, des sociologues, des spécialistes des sciences de l'éducation depuis au moins " les années 68 " - regards critiques dont la production doit être replacée dans son contexte pour être réellement appréciée -, il apparaît que l'inscription de tels regards dans la longue durée pose des questions récurrentes. C'est dire que si la vision critique (plus ou moins polémique) de l'école et de la famille comme lieux d'aliénation ou de domestication, depuis l'image du lycée-caserne jusqu'à la notion de " police des familles "1 ${ }^{1}$ est contextualisable et même datable, l'interrogation sur la place de la violence dans le système éducatif global reste une constante : les représentations se modifient, le degré de tolérance évolue, mais le soupçon demeure. Au cœur du système éducatif, dans la famille comme dans le système scolaire, est en effet posée en permanence la question de l'autorité, de son respect ou de sa transgression par rapport aux normes en vigueur. Là comme ailleurs, le sociétal et le légal sont en désaccord, n'allant pas toujours nécessairement dans le même sens. L'école, en tant qu'institution, constitue un reflet particulièrement intéressant de l'expression de sentiments divers (nostalgie, regrets, colère, dénonciation, etc.) face à la supposée inadéquation de

1 Pour reprendre le titre du classique de Jacques Donzelot, La Police des familles, Paris, Éditions de Minuit, 1977. 
ses lois d'autorité lorsqu'elle est confrontée à des générations juvéniles, productrices par nature de désordres.

$\mathrm{Au}$-delà des distinctions de tous ordres (types d'établissement, nature des cours dispensés, répartition géographique, composition sociale des élèves, etc.) qui font de l'enseignement secondaire un ensemble composite et disparate ${ }^{2}$, il apparaît que cet ordre d'enseignement reste défini, tout au long de la période envisagée ici (mais le propos est valable pour l'amont comme pour l'aval, depuis la création des lycées impériaux en 1802 jusqu'au lendemain de la Seconde Guerre mondiale), par la formule proposée par François Guizot dès 1816 : l'enseignement secondaire s'adresse aux " hommes qui sont destinés à avoir du loisir et de l'aisance, ou qui embrassent des professions libérales d'un ordre plus élevé, telles que le commerce, les lettres, etc. ${ }^{3}$. Vingt ans plus tard, le ministre de l'Instruction publique Pelet de la Lozère ne dit rien d'autre lorsqu'il définit l'instruction secondaire comme " destinée à cette partie de la jeunesse que sa position sociale ou ses talents appellent à exercer la plus grande influence sur l'avenir de notre patrie $"^{4}$. Pôle de fixation, le collège ou le lycée est donc appelé à maintenir et même à accentuer une sédentarisation sociale d'autant plus souhaitée que s'épanouit au même moment le discours sur la mobilité géographique et sociale et ses conséquences néfastes.

Entre une école primaire progressivement prise en charge par l'État, de la loi Guizot aux lois Ferry, et généralisée à l'ensemble de la population scolarisable, et un enseignement supérieur qui demeure le dispensateur de formations professionnelles spécifiques (droit, médecine, grandes écoles) ou de formations académiques ouvertes tant à de futurs professionnels qu'à un public d'amateurs (lettres, sciences, Collège de France), l'enseignement secondaire est le maillon central investi par les classes sociales suffisamment aisées pour jouer le jeu d'une réussite individuelle, et éventuellement d'une promotion sociale, facilitée par l'acquisition d'une culture commune aux élites : les humanités. Non sans inquiétude, du reste : comme l'affirme très clairement un rapport du Conseil

2 Sur l'enseignement secondaire, voir, au sein d'une foisonnante production, Maurice Gontard, L'Enseignement secondaire en France de la fin de l'Ancien Régime à la loi Falloux, 1750-1850, Aix-en-Provence, Edisud, 1984 ; Marie-Madeleine Compère, Du collège au lycée (1500-1850). Généalogie de l'enseignement secondaire français, Paris, Gallimard, 1985 (coll. Archives) ; Pierre Caspard, Jean-Noël Luc et Philippe Savoie (dir.), Lycées, lycéens, lycéennes. Deux siècles d'histoire, Lyon, INRP, 2005.

3 François Guizot, Essai sur l'histoire et sur l'état actuel de l'instruction publique en France, Paris, 1816, p. 2-3. Rappelons la première grande synthèse due à Georges Weil, Histoire de l'enseignement secondaire en France (1802-1920), Paris, 1921.

4 Pelet de la Lozère, "Circulaire aux recteurs, ayant pour objet de leur demander un Rapport exact sur la situation des établissements d'instruction secondaire de leur académie ", 7 juillet 1836. 
royal de l'Instruction publique de 1821 - la période est marquée par un certain nombre de révoltes de collégiens -, les collèges royaux dispensent des "idées qui ne sauraient devenir générales, sans devenir dangereuses par leur universalité même ${ }^{5}$. Est clairement mise en accusation la tentation de ces établissements (et de leurs directeurs comme de leurs enseignants, soumis dans les premiers temps de la Restauration à une assez sévère épuration, puis à une stricte surveillance) d'encourager le désir d'ascension sociale chez une catégorie d'individus appelés de fait à devenir des déclassés ; or le thème du déclassement social et de ses effets politiques ne cesse de hanter la société française tout au long du XIX siècle, la littérature (Balzac, Stendhal, Flaubert) s'en faisant largement l'écho. C'est aussi pourquoi, malgré la volonté, annihilée par de multiples résistances, de réformer le contenu de cet enseignement secondaire, le modèle humaniste hérité de l'Ancien Régime demeure longtemps indépassable. Qu'en est-il du modèle disciplinaire ?

À en croire le Michel Foucault de 1970, réfléchissant dans un contexte politique et culturel marqué par un rejet violent et assez largement partagé de l'école républicaine, accusée de reproduire les inégalités sociales, la concomitance entre le développement de l'enseignement secondaire et celui du suffrage, jusqu'à son universalisation, ne relève pas du hasard : "Le secondaire, s'épanouissant dans la philosophie, assurait la formation d'une élite qui devait compenser le suffrage universel, en guider l'usage, en limiter les abus. Il s'agissait de constituer, aux lieu et place d'un luthéranisme en défaut, une conscience politico-morale. Une garde nationale des consciences " ${ }^{6}$. L'analyse n'est, au fond, pas très différente de celle d'un Guizot. De cette approche des objectifs du collège découle la conséquence qui demeure l'apport le plus célèbre de la pensée foucaldienne à l'appréhension de la société post-révolutionnaire : l'école en général, et le collège en particulier, participent de cette politique d'enfermement illustrée également par l'hôpital, la prison, l'usine. Ces thèses ont été largement commentées, débattues et combattues, saluées et repoussées. Concernant le seul cas du collège, la théorie du " surveiller et punir " s'avère toujours une entrée intéressante pour analyser le mode de fonctionnement d'une institution dont l'élitisme proclamé ne peut faire l'économie d'une réflexion sur la notion de protection : protéger les collégiens ou les lycéens de l'intrusion de poisons pour l'esprit venus de l'extérieur ; se protéger

5 Archives nationales (désormais $\mathrm{AN}$ ), $\mathrm{AJ}^{16} / 884$, Écoles secondaires ou Écoles spéciales pour les classes de la société qui se livrent aux professions industrielles et manufacturières, rapport du Conseil royal de l'Instruction publique, 25 janvier 1821.

6 Michel Foucault, "Le piège de Vincennes ", entretien avec P. Loriot, Le Nouvel Observateur, $\mathrm{n}^{\circ} 274$, 9-15 février 1970, repris dans Dits et écrits, t. II, 1970-1975, Paris, Gallimard, 1994, p. 69. 
face à des cohortes de jeunes hommes dont la solidarité générationnelle et sociale est d'autant plus forte que le temps de l'enseignement secondaire est le temps de l'adolescence. Le développement des discours de toutes provenances (religieuse, juridique, médicale, psychologique, politique, etc., sans omettre la littérature dans son acception la plus large) sur l'adolescence comme temps de crise(s) est parallèle au développement de l'enseignement secondaire : si le terme reste plutôt associé à " un âge de classe " dans le premier XIX ${ }^{\mathrm{e}}$ siècle, avant de devenir dans les dernières décennies de ce même XIX ${ }^{e}$ siècle le substantif désignant " une classe d'âge " ${ }^{7}$, il est dès l'origine chargé de menaces. L'adolescent menaçant ne prend pourtant pas uniquement la figure du jeune bourgeois en rupture momentanée avec les valeurs transmises par son milieu socio-culturel : l'archive témoigne, plus abondamment qu'on le prétend, de la menace incarnée par une autre adolescence, populaire, rurale ou urbaine, parfois marginale et vagabonde ${ }^{8}$.

\section{I - Un conflit de sensibilités ? Autorité, société et génération}

Nous ne reviendrons pas ici sur l'arsenal réglementaire en usage dans les collèges, point déjà abordé ailleurs ${ }^{9}$. Mais on pourrait résumer ainsi la situation : le décalage entre la sévérité des règlements disciplinaires en vigueur, associée à la primauté rapidement accordée au régime de l'internat (considéré comme plus sûr que l'externat), et la progressive libéralisation des mœurs, en particulier celles qui sont relatives à la relation inter-générationnelle au sein de la famille bourgeoise,

7 Nous reprenons ici la distinction établie par Agnès Thiercé, Histoire de l'adolescence, 1850-1914, Paris, Belin, 1994.

8 Sur ce point, voir Jean-Claude Caron, Annie Stora-Lamarre et Jean-Jacques Yvorel (dir.), Les Âmes mal nées. Jeunesse et délinquance urbaine, XIXe-XXIe siècle, Àctes du colloque international de Besançon, 15-17 novembre 2006, à paraitre.

9 L'ouvrage pionnier en la matière est celui de Jeanne Contou, Les Punitions dans les lycées et collèges de l'Instruction publique en France (1814-1854). Approche historique d'une relation conflictuelle, thèse de III ${ }^{e}$ cycle sous la direction d'Antoine Léon, Université René Descartes - Paris V, 1980. Plus récent et marqué par l'influence de M. Foucault, Eirick Prairat, Éduquer et punir. Généalogie du discours psychologique, Nancy, Presses universitaires de Nancy, 1994. Nous nous permettons également de renvoyer le lecteur à Jean-Claude Caron, " Maintenir l'ordre dans les collèges et les lycées : théories et pratiques disciplinaires dans l'enseignement secondaire français (1815-1870) ", in Jean-Pierre Bardet, Jean-Noël Luc, Isabelle Robin-Romero, Catherine Rollet (dir.), Lorsque l'enfant grandit. Entre dépendance et autonomie, Paris, Presses de l'Université de Paris-Sorbonne, 2002, pp. 605-617 ; "Gouverner et sanctionner les jeunes élites. La grande enquête disciplinaire de 1853-1854 dans les lycées ", in Pierre Caspard, Jean-Noël Luc et Philippe Savoie (dir.), Lycées, lycéens, lycéennes, op. cit., p. 381-395 ; et, pour une approche plus large, "Les jeunes à l'école. Collégiens et lycéens en France et en Europe (fin XVIII ${ }^{e}$-fin XIX ${ }^{e}$ siècle) ", in Giovani Levi et Jean-Claude Schmitt (dir.), Histoire des jeunes en Occident, Paris, Seuil, 1996, t. 2, p. 143-207; À l'école de la violence. Châtiments et sévices dans l'institution scolaire au XIXe siècle, Paris, Aubier, 1999. 
est potentiellement producteur de conflit. Autrement dit, objet de démonstrations d'affection plus marquées de la part de ses parents, et en particulier de son géniteur, davantage autonome dans le quotidien de sa vie, le jeune homme de bonne famille s'émanciperait progressivement d'une tutelle disciplinaire fondée sur la soumission absolue à la puissance paternelle, telle que reconnue par le Code civil. Émancipation non pas nécessairement forgée contre la volonté paternelle, mais avec, sinon l'assentiment, du moins l'accord tacite d'un père converti à un rapport plus affectueux avec celui qui lui succédera. La thèse, fragile, est énoncée en particulier par Victor de Laprade et par Mgr Dupanloup. L'un et l'autre, chacun appuyé sur un modèle pédagogique propre (Rousseau, Fénelon), dénoncent, pour des raisons différentes, les effets néfastes de l'éducation publique. Depuis 1830 au moins, le camp clérical et, souvent, conservateur mène une croisade pour la liberté de l'enseignement secondaire - dont on sait l'aboutissement en 1850, avec la loi Falloux - dont le véritable héraut est Charles de Montalembert. La guerre scolaire des années 1830-1840 se focalise en grande partie sur le régime disciplinaire des collèges publics, accusés de produire des êtres irréligieux et revendicatifs quant à leur position sociale. La dénonciation de l'impiété de l'Université en général, et des établissements secondaires en particulier, niveau le plus sensible dans ce combat pour le contrôle des élites sociales en devenir, atteint alors son paroxysme. Derrière les noms célèbres incarnant cette véritable croisade anti-universitaire (Lamennais, Lacordaire, Montalembert), on trouve des seconds couteaux, plus déterminés encore, comme l'abbé Masson, ancien aumônier du Bon Pasteur et auteur du Miroir des collèges ou les vices effrayants de l'éducation universitaire sous le triple rapport du physique, de l'intelligence et du moral ${ }^{10}$. Cette dénonciation globale, fondée à la fois sur l'immoralité régnante, les pratiques pédagogiques et les conditions matérielles d'accueil des collégiens, témoigne, au-delà de son caractère militant et donc outrancier, de la place d'un argumentaire fondé sur la mobilisation de la sensibilité des parents, notamment sur la dureté du régime disciplinaire ${ }^{11}$. C'est précisément sur ce point qu'entend en bonne partie se fonder Mgr Dupanloup qui, dans de nombreux textes publiés des années 1840 aux années 1870, se pose en champion de la cause anti-universitaire ${ }^{12}$. Hostile au repli de l'aristocratie sur une éducation privée (préceptorale), il milite pour la liberté de l'enseignement secondaire, afin

10 Paris, Jacques Lecoffre et Cie, 1847.

11 Voir aussi la question des horaires imposés et de la répartition des séquences dans la journée : cf. Marie-Madeleine Compère (dir.), Histoire du temps scolaire en Europe, Paris, INRP, 1997.

Voir en particulier De l'éducation, Orléans, Gatineau,1850 ; De l'enfant, C. Douniol, 1869. 
de combattre "le soulèvement des classes inférieures contre les classes élevées et contre les supériorités sociales, c'est-à-dire le renversement prochain de tout ordre, de tout respect, de toute autorité, de toute hiérarchie, et par conséquent de toute société "13. Le collège est conçu comme "le noviciat du monde "14.

La position de Victor de Laprade, hostile à l'Université et à l'Instruction publique en général, rejoint par certains points celle de Mgr Dupanloup, tout en s'accompagnant d'une attaque conjoncturelle contre le Second Empire. Partisan d'une stricte hiérarchie sociale, le polygraphe Laprade critique de manière virulente ("l'éducation homicide") à la fois la fonction des lycées impériaux (produire "ce qui convient au césarisme et à la démocratie autoritaire : une population de fonctionnaires, je ne dis pas dévoués, mais dociles jusqu'aux dernières limites de la soumission " ${ }^{15}$ ) et, de manière beaucoup plus structurelle, faisant en quelque sorte le bilan de l'organisation de l'enseignement secondaire dans la première moitié du siècle, le collège comme couvent, caserne, prison, hôpital, évoquant même " ces bagnes de l'enfance qu'on appelle lycées, collèges ou séminaires " ${ }^{16}$. "Dressage violent et mécanique ", " éducation meurtrière du corps ", devoirs excessifs, production d'une race étiolée et efféminée, voire de cas de folie ${ }^{17}$ : la critique est radicale. Son caractère excessif ne doit pas dissimuler deux points importants : la première est que Laprade entend placer sa cause sur le terrain de la défense " des droits de l'enfant "; la seconde est que le débat suscité par ses propos rencontre des positions aussi radicales, émanant par exemple d'un Champfleury, d'un Chasles, d'un Michelet, d'un Sarcey, d'un Balzac, d'un Du Camp, d'un Hugo ou d'un Vallès. Longue serait la liste des "victimes " d'un enseignement secondaire ayant laissé dans le corps et plus encore dans l'esprit des meurtrissures indélébiles, remontant à la surface longtemps après, dans l'écriture mémorielle ou romanesque.

Même si l'on se doit de relativiser ces témoignages (il en existe de très favorables au temps des " années-collège "), ils ne peuvent être écartés sans tenir compte de l'existence d'un écart grandissant entre les sensibilités éducatives des différentes générations. C'est ce dont témoigne, sur le registre du constat plus que du regret, le directeur de la prestigieuse institution Sainte-Barbe, Labrouste, lorsque, confronté à la révolte de ces jeunes élites, il affirme : "la liberté dont 
leurs familles les laissent jouir leur rend fort lourd le joug de la discipline " ${ }^{18}$. On évoquera naturellement ici la construction d'un argumentaire visant avant tout à dédouaner sa responsabilité, ce dont témoigne précisément la récurrence de l'argument, largement utilisé par les chefs d'établissement confrontés à l'émeute collégienne. Mais cette récurrence prend aussi la signification d'une perception partagée : famille et école produisent deux modèles éducatifs et disciplinaires qui s'éloignent, voire s'opposent, dans les attentes et les contraintes ${ }^{19}$. Maxime Du Camp ne dit pas autre chose lorsque, se laissant aller à une critique en règle de ses années de collège, il explique "l'esprit d'insurrection " qui y règne par le régime disciplinaire inadapté en vigueur dans ces établissements. Lui-même renvoyé de Louis-le-Grand, puis de Saint-Louis, il dresse un bilan entièrement négatif de cette période de sa vie, au nom d'un individualisme non reconnu, d'une sensibilité assez partagée par les membres de sa classe sociale (qualifions-les de "bourgeois "), qui ne peuvent s'accommoder de la brutalité et de la vulgarité de l'environnement collégien : "Cette règle brutale, uniforme pour cinq cents caractères différents, la tristesse des cours entourées de hautes murailles et semblables aux préaux des prisons, la grossièreté, pour ne pas dire plus, des garçons qui nous servaient, la saleté des quartiers et des classes, l'aspect immonde de certains endroits où l'on ne pouvait se dispenser d'aller, l'odeur des réfectoires, la sévérité chicanière des maîtres d'étude, l'ironie des enfants qui s'efforcent à se moquer de tout bon sentiment, l'absence de liberté, l'oppression des individualités qui se redressent contre une domination systématique, ont fait pour moi du collège un enfer où j'ai toujours lutté et où j'ai toujours été vaincu ${ }^{20}$. Radicale, la critique n'est pourtant pas isolée, qui dit le hiatus entre une population visiblement consciente des égards dus à son origine sociale et le traitement carcéral infligé par et dans l'institution collégienne. Et le nombre élevé de révoltes touchant les collèges ou lycées publics dans les années 1815-1848 ne cesse d'alimenter un débat qui prend très vite une tournure politique.

$18 \mathrm{AN}, \mathrm{F}^{17}$ 8845, rapport au ministre de l'Instruction publique, 21 janvier 1847.

19 Sur l'éducation familiale et les relations intergénérationnelles, deux références : Linda A. Pollock, Forgotten Children. Parent-Child Relations from 1500 to 1900, Cambridge, Cambridge University Press, 1983 ; Dariah Vassigh, Les Relations adultes-enfants dans la seconde moitié du XIXe siècle (1850-1914). Étude discursive des écrits autobiographiques, éducatifs, juridiques et médico-légaux relatifs à cette question, thèse de doctorat d'histoire, sous la direction de Michelle Perrot, Paris VII, 1996.

Maxime Du Camp, Souvenirs littéraires, Paris, Aubier, 1994, p. 114. 


\section{II - Perception des révoltes collégiennes}

La multiplication des révoltes collégiennes sous la Restauration relève d'une causalité relativement homogène, formulable ainsi : à partir du moment où, jusqu'en 1828 au moins, il existe une situation de concurrence entre, d'un côté, des établissements secondaires dirigés par des religieux ou des petits séminaires faisant de facto fonction de collèges et, de l'autre, des collèges royaux, le choix d'un type d'établissement effectué par les parents relève souvent d'une position politique. Placer son fils dans un collège public, même dirigé par un abbé, même pourvu d'enseignants issus du clergé, cela peut prendre, en dehors d'un aspect pratique à ne pas négliger (la proximité géographique), une signification politique, comme en témoigne par exemple le cas du duc d'Orléans, qui scolarise ses fils au collège Henri IV. De manière assez logique, les collèges royaux tendent à devenir, avec les facultés, des lieux de résistance à la cléricalisation de l'Université. Il n'est donc pas surprenant que les désordres collectifs qui touchent les établissements secondaires sous la Restauration soient en grande partie localisés dans ces collèges où s'agglomère une jeunesse "patriote " - pour reprendre le vocabulaire alors en vogue -, qui affirme plus ou moins ostensiblement son libéralisme ou son bonapartisme et, davantage encore, son voltairianisme et son anticléricalisme. Les témoignages surabondent, pour les années 1820-1830, sur le verbe et l'acte antireligieux dans les collèges royaux, avec des formes variées de réticence, et parfois de résistance, à l'enseignement et à la pratique de la religion, allant, quoique rarement, jusqu'à la profanation d'objets sacrés, comme l'hostie.

Le recensement des établissements étroitement surveillés à l'époque témoigne de la prééminence des grands établissements parisiens, quel que soit leur statut : Louis-le-Grand, Henri IV, Charlemagne, Saint-Louis, Bourbon, mais aussi Stanislas, Sainte-Barbe, sont l'objet de fréquents rapports concernant la discipline au sens large du mot, incluant aussi bien la surveillance du corps administratif et du corps enseignant que celle des collégiens ${ }^{21}$. Pour autant, les autres types d'établissement ne sont pas en reste : recteurs et inspecteurs s'intéressent également

21 Voir par exemple AN, $\mathrm{AJ}^{16}$ 50-58, rectorat de Paris, collèges et lycées royaux, 1808-1849. 
aux collèges communaux ${ }^{22}$, ainsi qu'aux institutions et pensions ${ }^{23}$. Si les sources relatives aux maisons d'enseignement congréganistes qui accueillent des jeunes filles sont rares, elles sont plus nombreuses pour celles accueillant des jeunes garçons, ou des adolescents recevant, par exemple, une formation professionnelle : on peut ainsi citer l'Institut Saint-Nicolas, en précisant que les désordres constatés ne concernent pas des faits de révolte ou de rébellion, mais des mauvais traitements infligés aux élèves ${ }^{24}$. Il en est de même pour les établissements tenus par des jésuites : s'ils sont effectivement sujets à controverse, cela ne porte pas sur des cas de révoltes juvéniles, mais sur la présence même des jésuites en France et sur leurs méthodes disciplinaires (le Ratio studiorum) ${ }^{25}$.

Les célèbres " Bulletins de Paris ", qui donnent un état journalier des rapports de police relatifs aux désordres affectant la capitale, montrent également la place des " jeunes gens " comme catégorie soumise à un regard particulièrement attentif : la formule désigne aussi bien de jeunes calicots, de jeunes élites professionnelles, de jeunes militaires, que des collégiens et lycéens ou des étudiants ${ }^{26}$. Ces derniers, ainsi que les élèves des grandes écoles, sont particulièrement surveillés du point de vue de leur accointance supposée avec l'opposition libérale en général, et avec les sociétés secrètes, dont la Charbonnerie, en particulier. Plus jeunes, moins libres de leurs mouvements, les collégiens sont, quant à eux, essentiellement observés dans le cadre de leur établissement, beaucoup moins dans l'espace public. Du reste, la terminologie utilisée par la police pour désigner les fauteurs de troubles juvéniles ne permet pas toujours de catégoriser précisément ceux-ci : les "jeunes gens " désignent à l'évidence des ressortissants de classes moyennes, sans que l'on puisse parfois aller plus avant dans la précision. Mais, à l'occasion, la précision attendue est là, et des collégiens sont nommément désignés, ainsi que la nature du trouble

22 AN, $\mathrm{F}^{17}$ 8145-8164, collèges communaux, classement par académie, 1808-1848.

23 Nombreux exemples dans AN, $\mathrm{F}^{17}$ 8833-8861 ; voir par exemple $\mathrm{F}^{17}$ 8838-8841, institutions et pensions, état général par académie et par année, rapports des recteurs, 1833-1849; $\mathrm{F}^{17} 8842$, idem, rapports des inspecteurs généraux, 1810-1824. Sur le personnel universitaire en poste dans les collèges et les lycées, on se reportera au classique Paul Gerbod, La Condition universitaire en France au XIX ${ }^{e}$ siècle, Paris, Presses universitaires de France, 1965, complété par La Vie quotidienne dans les collèges et les lycées au XIXe siècle, Paris, Hachette, 1968. Plus récent, Philippe Savoie, Les enseignants du secondaire, XIX ${ }^{e}$-XX $X^{e}$ siècles. Le corps, le métier, les carrières, 1802-1914, Paris, INRP/Économica, 2000.

24 Voir par exemple AN, $F^{17}$ 12451-12479, en particulier 12465, Institut Saint-Nicolas. Historique, statuts, affaires disciplinaires et rapports d'inspection, 1838-1859.

25 John W. Padberg, Colleges in controversy. The Jesuit Schools in France from Revival to Suppression, 1815-1880, Cambridge, Harvard University Press, 1969 ; Pierre Michel, Le Mythe jésuite de Béranger à Michelet, Paris, PUF, 1992.

AN, F 3874-3893, Police générale, Bulletins de Paris, 1819-1846. 
à l'ordre public dont ils sont accusés : par exemple, chansons révolutionnaires et rébellion pour les élèves du collège royal de Versailles en $1833^{27}$.

Les mots utilisés illustrent bien cette volonté sans cesse réaffirmée par le pouvoir, par-delà les changements de régime politique, de surveiller "l'esprit public " ${ }^{28}$. Le plus fréquemment, c'est bien d'" affaires disciplinaires " qu'il est question, simple atteinte aux règlements en vigueur dans les établissements ${ }^{29}$. Mais d'autres mots illustrent la peur particulière générée par cette politisation juvénile des classes moyennes, élites en devenir, dont les révoltes au collège sont perçues aussi bien comme un simple comportement générationnel (l'adolescence comme temps de la contestation) que comme une grave remise en cause de l'ordre social : ce sont bien des " insurrections dans les collèges et les pensions " que la police et la justice entendent traiter sous la Restauration ${ }^{30}$, en relation avec la grande vague protestataire des facultés, des casernes (pensons aux sergents de La Rochelle) et, plus largement, des sociétés secrètes, alors qu'en 1847 on parle de "désordres graves aux collèges royaux de Henri IV et Sainte-Barbe "31 et en 1849 de "troubles " dans les lycées parisiens ${ }^{32}$. L'administration de l'Instruction publique n'est pas en reste : les recteurs sont sommés de produire des rapports de surveillance sur tous les établissements d'enseignement secondaire de leur académie ${ }^{33}$.

Encore convient-il de réfléchir à deux aspects du traitement des révoltes collégiennes. Le premier concerne leur dénomination : celle-ci est attribuée par la source en fonction de paramètres qui peuvent aussi bien émaner du producteur de la source que de l'archiviste chargé de son classement. Il faut donc relativiser les catégories attribuées et, en reprenant les dossiers, systématiquement comparer la nature de ces révoltes. On s'aperçoit alors que l'enjeu dénominatif est essentiel et cache des événements qui, bien que parfois très proches, ne sont

$27 \quad \mathrm{AN}, \mathrm{F}^{7}$ 6783, dossier 3.

28 Pierre Karila-Cohen, "L'État des esprits ". L'administration et l'observation de l'opinion départementale en France sous la monarchie constitutionnelle (1814-1848), thèse de doctorat, sous la direction d'Alain Corbin, $1^{\text {er }}$ décembre 2003, Université de Paris I-Panthéon-Sorbonne.

29 AN, $\mathrm{F}^{17}$ 7287-7289, collèges royaux, affaires disciplinaires, 1818-1854; $\mathrm{F}^{17}$ 8109-8135, affaires générales des collèges classées par académie, 1810-1855 ; nombreux exemples également dans $\mathrm{F}^{17} 8846$-8847 pour la Seconde République, le Second Empire et les débuts de la Troisième République ; F ${ }^{17} 8858$, affaires disciplinaires, 1817-1849.

$30 \mathrm{AN}, \mathrm{F}^{7}$ 6903, insurrections dans les collèges et les pensions, 1820-1825.

$31 \mathrm{AN}, \mathrm{F}^{17} 8845$.

$32 \mathrm{AN}, \mathrm{F}^{17}$ 6812, troubles dans les lycées parisiens, 1849.

$33 \mathrm{AN}, \mathrm{F}^{17}$ 2668-2675, renseignements sur fonctionnaires de l'enseignement et collèges royaux ; voir, par exemple, $\mathrm{F}^{17} 2670$, notes sur le collège royal de Cahors et autres collèges, $1836 ; \mathrm{F}^{17} 2671$, académies de Douai, Grenoble, Lyon, 1836 ; $\mathrm{F}^{17} 2673$, académie de la Seine, 1836. 
pas nommés de la même manière. Pour le dire autrement, la qualification relève bien de l'ordre du politique, surtout lorsque les révoltes collégiennes s'inscrivent dans un contexte contestataire, voire protestataire, plus général. C'est en particulier le cas dans les années 1819-1824 ou dans les premières années de la monarchie de Juillet. Le deuxième aspect qui mérite réflexion concerne l'économie globale de la violence dans les collèges et lycées. Il est certes loisible, et même utile, d'isoler les révoltes collégiennes, reproduisant ainsi le classement adopté par l'archive. Mais, outre que les dénominations adoptées peuvent être sujettes à caution, comme on vient de le voir, cet isolement ne permet pas d'appréhender dans sa globalité l'expression d'une violence dont la révolte - ici entendue dans un sens politique, pour la grande majorité des cas - n'est que la traduction la plus visible. Que faire, pour prendre une représentation se situant sur le registre de l'humour, de "l'émeute " écolière (entendue ici au sens de collégien, tout autant que d'élève) dessinée par Daumier en $1849^{34}$ ? Faut-il la considérer comme le signe d'une révolte adolescente s'exprimant par mille autres formes de résistance à la stricte discipline imposée par le règlement et ne relevant pas vraiment d'une lecture politique ? Mais ce refus d'une lecture politique n'est-il pas contredit par la représentation d'un peuple (incarné par le collégien) se révoltant contre ses maîtres (dont le représentant le plus contesté est le "pion "), objet de toutes les formes (nécessairement illégales) de contestation?

Le collège constitue bien une parodie de la société et de ses rapports sociaux, un lieu d'apprentissage à la fois des codes sociétaux, moraux et politiques, un microcosme social, certes tronqué, où des professeurs et des pions, immanquablement représentés comme des ratés de l'ascension sociale, dressent à l'obéissance, voire à la soumission, de futurs Joseph Prudhomme portant déjà les signes de leur appartenance à une certaine classe sociale : embonpoint menaçant, bonnets de nuit... " L'émeute " n'est au fond que la traduction, utilisée ici sur le registre comique, du droit à l'insurrection contre un gouvernement despotique ${ }^{35}$. La mémoire répulsive du collège est la chose la mieux partagée dans la France du $\mathrm{XIX}^{\mathrm{e}}$ siècle, autour d'un constat commun : le règne de la violence triomphe sous toutes ses formes, produisant dès lors une résistance multiforme qui débouche, à l'occasion, sur la révolte. Celle-ci est à la fois sociale et générationnelle, expression de ce hiatus entre deux formes éducatives qui tendent à s'éloigner l'une de l'autre. La

34 Daumier et l'Université. Professeurs et moutards. Préface, catalogue et notices par Raymond Picard, [Paris], Michèle Trinckvel, 1992. 
figure encore trop mal connue du maître d'étude cristallise ce hiatus. Une majorité des révoltes collégiennes recensées sous la Restauration et la monarchie de Juillet sont provoquées par l'affrontement entre le maitre d'étude, souvent présenté comme la figure type du raté ou du déclassé social dans la littérature romanesque (voir Daudet et Vallès) ou mémorielle, et de jeunes élites habituées à traiter avec la domesticité familiale. L'archive regorge d'affaires, individuelles ou collectives, témoignant de la virulence de cette opposition entre deux catégories que séparent moins une différence de génération (les maîtres d'étude sont généralement jeunes, encore étudiants ou, pour reprendre une formule de l'époque, "étudiants de dix ans ", c'est-à-dire s'accrochant à un statut social que dément l'échec dans les études suivies sur une longue durée) qu'une différence sociale. La rigueur avec laquelle le maître d'étude applique le règlement, menacé qu'il est de renvoi en cas d'insubordination des collégiens, entraîne des pratiques vindicatoires de toutes natures, qui dégénèrent à l'occasion en révolte collective. La haine qui émane des collégiens ou des lycéens envers ce détenteur d'une autorité dont la légitimité n'est pas reconnue est surtout le fait des élèves à la fois les plus âgés et les plus brillants, ceux des classes préparatoires. C'est pourquoi, prenant conscience de cette situation, le ministre de l'Instruction publique ordonne en 1845 aux proviseurs des lycées Louis-le-Grand, Saint-Louis et Henri IV, de séparer cette élite des autres élèves et de ne les confier qu'à des maîtres d'étude qui soient gradués en sciences. Deux des trois proviseurs répondent que telle est déjà leur pratique, qui, visant à atténuer le décalage intellectuel, culturel et social entre élèves et maitre d'étude, entend également agir sur le maintien de l'ordre ${ }^{36}$.

\section{III - La révolte collégienne, une forme politique ?}

Simple chahut, contestation plus ou moins violente et ouverte du règlement, rébellion collective parfois qualifiée d'insurrection et traitée comme telle, la gamme des désordres au collège est large. Celle des réactions des autorités ne l'est pas moins, depuis une certaine tolérance envers des formes attendues, quasiment traditionnelles, de révolte juvénile caractérisant à la fois un groupe social et un groupe générationnel, jusqu'à une violente répression, incluant intervention de forces de l'ordre, exclusion définitive et, parfois, sanction pénale. Figure la plus courante, et de fait la plus tolérée, du désordre collégien, les violences individuelles ou collectives entre élèves ne sont révélées que lorsqu'elles dépassent la limite d'un 
tolérable lui-même très variable. La cour de récréation demeure le champ clos de batailles d'honneur ou de suprématie, codifiées, attendues, et rarement dénoncées par les autorités. Quant à la révolte collégienne, étant prévisible, ce n'est pas tant sa manifestation que ses causes, ses modes et ses limites qui sont scrutés et qui appellent dès lors une réponse modulée. Il ne s'agit pas ici, en effet, de châtier des paysans ou des ouvriers, mais de jeunes bourgeois dont les familles constituent une forme d'élite sociale et, accessoirement, la clientèle payante d'établissements qui se livrent à une redoutable concurrence : la connivence sociale l'emporte dans la très grande majorité des cas.

Peut-on quantifier les révoltes collégiennes sous la monarchie constitutionnelle (1815-1848), étudier leur répartition dans le temps et l'espace, en établir une typologie ? La quantification demeure difficile, sauf à dresser une liste de critères objectifs que la source fournirait. Ici, on conservera l'expression de révolte collégienne à des actes collectifs de désobéissance ayant entraîné des formes inhabituelles de gestion par les autorités universitaires, au-delà de celles que prévoient les règlements disciplinaires. Mais, selon les époques, la qualification même de " révolte " est attribuée à des mouvements collectifs de factures très diverses. Ce qui renvoie à la fois à leur distribution dans le temps et dans l'espace et à leur typologie. Une corrélation existe entre les grandes phases révolutionnaires ou insurrectionnelles $(1830,1832,1848)$ et l'agitation collégienne ou lycéenne. Mais la grande majorité des révoltes juvéniles se produisent en dehors de ces périodes, pour des motifs qui relèvent bien de tensions internes et non de l'influence du contexte politique. On remarque pourtant l'exception des années 1819-1824, où les collèges connaissent une vague de révoltes en phase avec le mouvement contestataire étudiant, à Paris, mais aussi à Grenoble ou à Montpellier ${ }^{37}$. On sait que ce mouvement contestataire touche, au-delà de la population étudiante et collégienne, une partie non négligeable de la jeunesse libérale, bonapartiste, voire républicaine. À Paris, ce sont surtout les collèges Henri IV et Louis-le-Grand qui sont touchés. L'Est (l'Alsace en particulier) et l'Ouest (la Bretagne, notamment), "bleus " tous deux, sont en pointe dans cette révolte collégienne. Mais le Midi, toulousain comme provençal, n'est pas en reste et le lycée de Marseille apparaît à la pointe de cette résistance collective, à défaut d'être globale : car il y a aussi des "blancs " dans ces établissements et les premiers désordres se produisent

37 Nous nous permettons de renvoyer à Jean-Claude Caron, Générations romantiques. Les étudiants de Paris et le quartier Latin, 1814-1851, Paris, A. Colin, 1991. 
souvent lorsque les deux camps s'affrontent ${ }^{38}$. Deux camps qui, dans les grandes villes en particulier, peuvent appartenir à deux établissements différents, chacun étant réputé pour sa " couleur " politique et religieuse : les incidents se produisant dès lors sur la scène publique, dans la rue, et non dans l'enceinte de l'établissement, leur publicité est assurée, comme l'attestent, par exemple, les bagarres qui opposent durant plusieurs jours en 1819, à Paris, des élèves d'un établissement tenu par des frères à ceux d'un établissement d'enseignement mutuel, dont on sait la réputation libérale ${ }^{39}$.

Les années 1815-1848 sont intéressantes en ce qu'elles permettent d'aborder la vie politique sous le registre de l'engagement générationnel. Ce sont bien des " jeunes gens " qui, étudiants, élèves de grandes écoles, collégiens, sont identifiés dans ces mouvements de révolte collective : quelle que soit par ailleurs la cause de ces derniers, l'implication de ces mineurs, au point de vue juridique comme au point de vue civique, apparaît comme une nouveauté, redoutée par les autorités, saluée par l'opposition, quitte à " politiser " ce qui ne relève parfois que d'une contestation disciplinaire. Car, au-delà de cette première explication, la seconde, qui renforce par ailleurs la première, tient essentiellement au refus des obligations religieuses, cristallisé sur l'assistance imposée à la messe, telle qu'elle est définie dans les règlements des collèges. L'irréligion des collégiens est trop souvent soulignée pour ne pas correspondre à un certain degré de réalité. Accusé de ne pas tenir ses pensionnaires, candidats aux concours des grandes écoles qui se livrent à toutes sortes de comportements immoraux aux dépens du voisinage (insultes, obscénités, chants politiques), le directeur d'une institution se défend en accusant ses élèves " qui se croient des étudiants et non plus des écoliers et dont les parents sont, trop souvent, assez aveugles pour demander qu'on ne s'occupe point de leçon de morale ; au point que le chef de l'institution en est réduit à faire une sorte de violence à ces jeunes gens pour les conduire à l'église, où l'on conçoit qu'ils ne se montrent pas d'une piété exemplaire "40. Là encore, on peut évoquer l'exemplarité du discours, pointant les responsabilités familiales dans les progrès de l'incroyance juvénile.

Ce plaidoyer pro domo illustre aussi, à sa manière, la concurrence entre deux conceptions (juvénocratie contre gérontocratie) d'une société fondée sur une hié-

38 Sur Marseille, Maurice Gontard (dir.), Histoire des lycées de Marseille, Aix-en-Provence, Edisud, 1982. Et du même, dans une perspective plus large, L'Enseignement secondaire en France de la fin de l'Ancien Régime à la loi Falloux, op. cit.

$39 \mathrm{AN}, \mathrm{AJ}^{16} 883$, lettre de Cuvier à l'inspecteur d'académie, 30 novembre 1819.

40 AN, $\mathrm{F}^{17} 8846$, affaire de l'institution Brion, mai 1845. Souligné dans le texte. 
rarchie parallèle, générationnelle et sociale, justifiant la détention de positions de pouvoir et d'autorité. En pointant l'irréligion des révoltés, l'administration des collèges royaux se débarrasse à bon compte de questions concernant sa propre responsabilité, en préférant mettre en avant les insuffisances morales et religieuses de l'éducation familiale. Mais cela permet également (pas toujours) de dépolitiser les motifs de ces révoltes : à tout prendre, mieux vaut des collégiens irréligieux qu'hostiles à la monarchie restaurée. De fait, dans la hiérarchie des causes morales de ces désordres, l'invocation très souvent prioritaire concerne l'absence de tout esprit religieux chez ces futures élites. Et la présence d'un clerc, par exemple un abbé, comme chef d'établissement, liée à celle d'un aumônier particulièrement tatillon sur les pratiques religieuses, font de ces dernières un élément déclencheur communément attesté : refus d'assister à la messe, ou plus souvent refus d'y chanter, voire entreprise de détournement du caractère sacré de la cérémonie par des attitudes ou des gestes témoignant du refus de la contrainte d'y assister. De tels comportements sont révélés par les récits de deux vagues de révoltes collégiennes concomitantes, en 1819 (Louis-le-Grand, Rennes, Nantes, La Flèche, Saint-Cyr, Périgueux) et 1822 (Nantes, Pontivy, Poitiers, Amiens, Toulouse, Henri IV) ${ }^{41}$. À Toulouse, par exemple, le refus des élèves de chanter les vêpres amène le proviseur à sévir, ce qui provoque par réaction une véritable mutinerie d'élèves se barricadant dans une salle d'étude ${ }^{42}$.

Mais, plus ou moins clairement énoncé, l'aspect politique de certaines de ces révoltes inquiète les autorités, confrontées par ailleurs à une contestation étudiante radicale avec la mort de l'étudiant en droit Nicolas Lallemand, tué en juin 1820 lors d'une manifestation de protestation contre la modification de la loi électorale ${ }^{43}$, et à une agitation bonapartiste dans certains régiments, en particulier dans le milieu des sous-officiers, jeunes hommes nourris de la geste napoléonienne qui n'hésitent pas à adhérer à la Charbonnerie. Aussi le portrait d'un des meneurs supposés de la révolte du collège de Rennes en 1819 soulève-t-il les inquiétudes du préfet de l'Ille-et-Vilaine : neveu d'un conseiller à la cour royale, petit-neveu de l'abbé Carron, futur évêque du Mans et soutien sans faille du Bon Pasteur, ce collégien est accusé d'avoir systématiquement refusé de chanter le Domine salvum fac regem, de persister à appeler lycée le collège et d'être l'auteur de deux textes intitu-

41 Voir les différents dossiers dans AN, $\mathrm{F}^{17} 7287$.

$42 \mathrm{AN}, \mathrm{F}^{17} 7287$, brouillon d'une lettre du président du Conseil royal de l'Instruction publique au ministre de l'Intérieur, 26 mars 1822.

43 Il s'agissait de l'adoption de la loi du " double vote ", qui permettait aux électeurs les plus imposés, donc les plus riches, de faire prévaloir leur point de vue. 
lés "Les lycéens de Louis-le-Grand aux lycéens de Rennes " - ce qui manifesterait une ébauche de réseau entre élites juvéniles de villes différentes - et "Association des bons enfants " ${ }^{44}$. Ces deux textes sont intéressants en ce qu'ils témoignent de l'existence, rarement matérialisée, d'une parole adolescente de nature politique : appel à la solidarité interne fondé sur la notion d'honneur et le rejet de la tyrannie, appel à la solidarité externe avec le rappel des mouvements affectant les collèges de Paris, Nantes, La Flèche et Saint-Cyr, appel à l'association, enfin, avec énoncé de procédures secrètes d'affiliation et de reconnaissance. Le désir d'intégration à la citoyenneté que traduisent des textes d'inspiration " patriote " (à mi-chemin entre bonapartisme et libéralisme) conduit aussi à s'interroger sur les processus de transmission de l'expérience politique, entre générations notamment, et sur les modes de diffusion d'une culture d'opposition ne disposant pas, a priori, d'organes publics. Decazes, en charge de l'Intérieur, prend l'affaire au sérieux, considérant que la simultanéité des mouvements dans plusieurs collèges ne relève pas du hasard, et pousse le préfet de l'Ille-et-Vilaine à entretenir " quelque intelligence dans l'intérieur du collège, et parmi les externes ", catégorie particulièrement surveillée, car redoutée en raison de sa circulation entre le collège et l'extérieur ${ }^{45}$.

La politisation de certaines de ces révoltes n'est pas nécessairement niée par les familles, qui, cependant, n'hésitent pas à prendre fait et cause pour leur progéniture. Il est en effet frappant de considérer le nombre et le contenu des lettres adressées au président du Conseil royal de l'Instruction publique, puis au ministre en charge du même département, par les pères d'élèves exclus de leur établissement ou menacés de l'être pour cause d'insubordination, de mutinerie ou de révolte. Loin de se cantonner au registre de la soumission et du pardon justifié par le jeune âge des mutins, ces lettres dénoncent, à l'occasion, l'administration des établissements, contestent les versions des événements données par les proviseurs, mettent en avant le principe de l'amalgame comme injuste. Missives redoutables par leur argumentaire très solidement construit, émanant d'hommes rompus aux arcanes de la chicane, portant parfois un nom illustre et apportant crédit au récit fait par leur enfant : ainsi Pierre-Alexandre du Sommerard, fils d'Alexandre du Sommerard, fondateur du Musée de Cluny et conseiller référendaire à la Cour des Comptes, écrit-il à son père pour contester la version de la révolte du collège d'Amiens, en 1828, qu'a établie le proviseur ${ }^{46}$. À la suite de 
graves incidents ayant touché en 1829 la pension Mayer, institution parisienne réputée qui accueille des élèves préparant le concours de l'École polytechnique, le directeur entend sévir en renvoyant en bloc les " meneurs ". Mais lorsque ceux-ci sont apparentés aux Salvandy ou s'appellent Gasparin, la mesure n'est pas aisée à appliquer ; au-delà de ces deux cas, les autres exclus - ou menacés de l'être - des établissements parisiens (soit dix-sept individus au total) sont fils d'un négociant lyonnais membre du conseil municipal, fils d'un avocat valenciennois, fils d'un ancien préfet, fils d'un juge de paix, fils d'un chef de bataillon, fils d'un maître de forges, fils d'un lieutenant du roi, commandant militaire à Besançon, etc. ${ }^{47}$ La plupart de ces exclusions furent, de fait, très temporaires ; a contrario, Vatimesnil décida de traduire Mayer, le directeur de l'institution, devant le conseil académique de la Seine, qui prononça une réprimande à son égard pour n'avoir pas su empêcher la révolte ${ }^{48}$. Et le directeur dut joindre sa prose à celle des parents des exclus - finalement tous réinscrits dans d'autres établissements parisiens - pour donner son accord à la levée des sanctions et dire qu'il souhaitait la réintégration de ces élèves dans les collèges de la capitale.

L'aspect politique n'est jamais loin dans ces procédures d'exclusion ou de réintégration : mêlé à une révolte touchant le collège royal de Marseille en juin 1830, un élève est réintégré dans celui d'Aix, car il appartient à une famille ruinée par la Révolution et est le neveu d'un avocat qui " professe les meilleurs principes sous tous les rapports " ${ }^{49}$. Au lendemain des Trois Glorieuses, c'est aussi la politique qui est mise en avant par le père d'un élève exclu du lycée de Rouen. Écrivant à Montalivet, il demande l'indulgence du ministre au nom du contexte politique : "L'impulsion extraordinaire donnée aux esprits par les glorieux événements de juillet a pénétré jusque dans l'intérieur des collèges. Des têtes jeunes et ardentes ont dû facilement en être troublées ". Son fils est finalement réintégré, mais comme externe ${ }^{50}$. L'indulgence est la même, en 1833, envers huit mutins du collège Saint-Louis, dont trois meneurs qualifiés de légitimistes : ceux-ci, qui ont pris un maître d'étude en otage et se sont barricadés dans leur quartier, auraient crié "Vive la Vendée ! Vive Henri V! " avant de se rendre. Le souvenir de l'expédition de la duchesse de Berry est encore très présent. Parmi ces huit exclus, figurent

\footnotetext{
$47 \mathrm{AN}, \mathrm{F}^{17}$ 7287, rapport du 8 avril 1829 et lettre au ministre de l'Instruction publique, 13 avril 1829.

$48 \mathrm{AN}, \mathrm{F}^{17} 7287$, conseil académique de la Seine, séance du 21 mai 1829.

$49 \mathrm{AN}, \mathrm{F}^{17}$ 7287, note à M. le conseiller Guéneau de Mussy, 6 juillet 1830 .

50 AN, $\mathrm{F}^{17} 7287$, lettre d'Henry Lefort, manufacturier, au ministre de l'Instruction publique, 27 avril 1831.
} 
le fils d'un ancien directeur des haras, le fils d'un député, le fils d'un professeur au Conservatoire des Arts et Métiers, le fils de l'ancien maire de Brive, etc. ${ }^{51}$. Le contexte politique est également invoqué en avril 1834 à l'occasion d'une révolte au collège de Nantes, dont le proviseur voit la cause dans " la fermentation qui agitait les têtes au dehors " ${ }^{52}$ et signale qu'un des mutins, âgé de seize ans, accusé d'avoir porté un ruban rouge à sa boutonnière (insigne notoirement républicain), aurait fait allusion aux événements parisiens. Le cas est comparable avec la révolte qui secoue au même moment le collège de Lyon, et que son proviseur attribue à "l'effet des sinistres événements dont Lyon fut le théâtre " ou encore au "retentissement de la guerre civile qui a désolé cette grande cité ${ }^{53}$. Là encore, les meneurs, âgés de 15 à 17 ans, sont soupçonnés de sympathies républicaines.

\section{IV - Trois cas de révolte radicale : Louis-le-Grand (1824), Amiens (1828), Sainte-Barbe (1844)}

Quelques exemples précis, sortant toutefois de l'ordinaire de la révolte par leur ampleur, permettent de mieux saisir les formes de la rébellion collégienne et de leur gestion par les autorités : ainsi de Louis-le-Grand en 1824, d'Amiens en 1828, de Sainte-Barbe dans les années 1840. Ce dernier établissement fut en effet touché à deux reprises $(1844,1847)$ par des révoltes de grande ampleur. Dans les trois cas, ce sont surtout les élèves les plus âgés qui sont accusés d'avoir été les promoteurs de la révolte, en particulier les élèves de la classe de philosophie et, plus encore, ceux qui suivent les classes préparatoires aux concours d'entrée dans les grandes écoles. Est également pointé le rôle spécifique des externes, qui sont accusés de faire entrer le désordre moral et politique dans l'établissement. Le détour par la morale s'impose d'autant plus que, assez fréquemment, les dossiers constitués sur les collégiens menacés d'exclusion mentionnent des " comportements immoraux " : en dehors de cas rares, et concernant uniquement des externes accusés d'avoir fréquenté des filles de mauvaise vie, sont dénoncés des sentiments " contre nature " qui auraient uni deux collégiens. La description qui est faite de ces comportements et la relative fréquence de leur mention incitent plutôt à parler d'amitié amoureuse, de sexualité de substitution entre internes,

51 AN, $\mathrm{F}^{17} 7287$, lettre du proviseur du collège royal de Saint-Louis au ministre de l'Instruction publique, sans date [9 février 1833]

52 AN, $\mathrm{F}^{17}$ 7287, lettre du proviseur au recteur de l'académie, 19 avril 1834.

$53 \mathrm{AN}, \mathrm{F}^{17} 7287$, lettre du proviseur au recteur de l'académie, 29 avril 1834. 
sur le registre de l'élection, réciproque ou non (il y a parfois une différence d'âge notable entre les deux " coupables "), plutôt que d'homosexualité. Il s'agit en tout cas de circonstances aggravantes, d'autant plus mentionnées qu'elles permettent de combler les vides des dossiers accusateurs et de faciliter l'exclusion des élèves concernés.

Autre point commun, à l'origine de ces révoltes violentes, le rejet des formes disciplinaires prévues par les règlements et du personnel qui se trouve en première ligne pour les appliquer : le censeur - fusible naturel pour préserver l'autorité du proviseur - et, davantage encore, le maître d'étude. À Amiens, c'est le manque d'autorité du censeur qui est stigmatisé. Cela pose, par ailleurs, la question de la relation entre les deux pôles de l'autorité bicéphale qui dirige tout établissement (proviseur/censeur) : nombre d'affaires mettent en avant la rivalité ou le désaccord opposant les deux individus, ce qui aurait favorisé la détérioration de la discipline. De même affirme-t-on souvent, comme dans les trois cas étudiés ici, que des signes avant-coureurs annonçaient l'imminence de l'explosion, mais que, faute d'unité entre les administrateurs, rien n'a été fait pour prendre des mesures préventives. À Amiens, le thème de la rumeur du " complot de soulèvement " 54 est mis en avant, ce qui sous-entend une organisation, une planification, la circulation de bruits, des avertissements non pris en compte, émanant en particulier des maîtres d'étude, sollicités comme informateurs privilégiés. L'aumônier est parfois également à l'origine de la révolte, notamment lorsqu'il applique à la lettre les obligations religieuses imposées aux collégiens. À Louis-le-Grand, le proviseur explique que des élèves " prétendent que je prépare la voie aux Jésuites, qu'on veut les plier à toutes sortes de pratiques religieuses, et qu'une révolte générale peut seule les sauver "55. Des voix jugées " discordantes " se font entendre aux vêpres, puis tous les élèves cessent de chanter à tous les offices. Plus grave, car on sort du religieux pour toucher au politique, le proviseur reconnaît que lors du Domine salvum fac regem, les élèves ne chantent pas le dernier mot et que, à l'occasion du banquet de la Saint-Charlemagne, ils ont gardé le silence lorsque un toast a été porté à la santé du roi. Enfin, ce sont parfois de simples domestiques qui, par leur comportement jugé grossier, provoquent des réactions violentes de ces jeunes élites.

L'aspect nocturne de ces révoltes est un élément essentiel : la révolte éclate avec la destruction des lanternes ou des quinquets, rendant beaucoup plus difficile la reconquête des quartiers insurgés. Labrouste, directeur de Sainte-Barbe, dit la

$54 \quad \mathrm{AN}, \mathrm{F}^{17} 7287$, lettre du recteur au ministre de l'Instruction publique, 27 février 1828. 
quasi impossibilité d'intervenir face à une révolte qui se déroule " par une obscurité complète, par une nuit sans lune, les lanternes brisées " - " au milieu des ténèbres ", ajoute l'inspecteur d'académie ${ }^{56}$. L'espace de la révolte est d'abord l'espace du barricadement, de la délimitation d'une zone en dehors de la soumission à la règle. La récurrence du mot de "barricade " (et de ses dérivés) pour désigner ce qui n'est en définitive, le plus souvent, qu'un simple amoncellement de matériaux divers souligne le lien que les administrateurs des collèges entendent établir avec l'émeute urbaine. L'accent est mis sur l'importance des dégradations matérielles, dûment chiffrées afin à la fois de mettre en avant la barbarie ou la sauvagerie des acteurs de la révolte et d'obtenir une réparation financière des autorités de tutelle : les inventaires dressés témoignent des usages barricadiers de tous les objets présents dans ces établissements, mais aussi des usages festifs des denrées alimentaires, une attention particulière étant portée à la consommation de vin et de liqueurs. La description de ces révoltes nocturnes évoque irrésistiblement le mode carnavalesque ou charivarique, une forme de défoulement brutal. Face à une appropriation de l'espace sonore par des " hurlements ", " vociférations " et autres chansons dites "obscènes " - sans que l'on sache s'il s'agit d'obscénité morale ou d'obscénité politique -, face à une gestuelle inquiétante, incluant parfois des danses, un directeur comme Labrouste utilise des expressions propres au registre de l'aliénisme (" exaltation furieuse ", " coupable frénésie ", " coupable folie ", " délire furieux ", " acte sauvage et extravagant ") ou de la métaphore hygiéniste (" fièvre contagieuse de l'émeute "), que l'on retrouve dans les discours de stigmatisation de l'insurrection populaire ${ }^{57}$. Il s'agit certes, pour le directeur, de détourner toute forme de mise en cause de sa responsabilité. Mais l'opposition reste tranchée entre l'extrême violence exercée par les révoltés sur l'environnement matériel et l'absolu respect de l'intégrité physique de tous les acteurs de ces révoltes : opposition qui apparait, en définitive, comme le meilleur marqueur de la spécificité sociale de la mutinerie collégienne et de son traitement politique.

Lorsque le degré d'intensité de la révolte rend impossible toute reprise en main de la situation en interne, on fait appel aux forces en charge du maintien de l'ordre public, autorisées à pénétrer dans les établissements et à reprendre les bâtiments par la force - force dont l'usage est toutefois strictement codifié pour empêcher tout dérapage : cette révolte de l'élite du savoir destinée à devenir l'élite du pouvoir social, économique, politique, ne peut se gérer comme une révolte de prisonniers,

56 AN, F ${ }^{17}$ 8845, affaire Sainte-Barbe, rapport en date du 26 mars 1844.

57 Id. 
de soldats ou même de pensionnaires de Mettray. Entrent alors en action sergents de ville, gardes municipaux - dont le commandant est par ailleurs père d'un élève de Sainte-Barbe -, gardes nationaux ou gendarmes, voire, dans les cas jugés les plus graves, troupes de ligne ; et, malgré quelques affirmations contraires, il semble qu'aucun collégien n'ait été blessé à coup de sabre ou de baïonnette. En revanche, dans le cas de la révolte de Sainte-Barbe, la décision prise par Labrouste de vider complètement son établissement en pleine nuit en renvoyant la totalité des pensionnaires - soit quelque 130 élèves -, y compris ceux qui n'ont pas de famille à Paris, est très sévèrement jugée par le Conseil royal de l'Instruction publique, tout comme est mal perçu leur regroupement préalable dans la cour sous la garde de soldats ayant mis la baïonnette au fusil. Le conflit se déplace de la procédure habituelle (exclusion des "fautifs " puis, à la demande des familles, intervention d’intermédiaires - député, évêque, prêtre, directeur de pension, etc. - pour demander et le plus souvent obtenir une atténuation de la sanction) vers un débat sur la " mesure inouïe " ou le " fait incroyable " qu'a constitué cette expulsion générale nocturne ${ }^{58}$. Le procès des insurgés tend à devenir celui de Labrouste, d'autant que le débat sur le monopole universitaire est vif et que les partisans de la liberté de l'enseignement profitent des événements pour critiquer l'immoralité et l'absence d'autorité qui règnent dans les lycées. Ulcéré et amer, Labrouste se dit " assailli de prières, de sollicitations, de menaces " émanant des familles dont le fils a été renvoyé, et doit affronter le conseil d'administration de son établissement, dans lequel siègent le comte Dumas, aide de camp du roi, des députés comme Ganneron et Vavin, des personnalités comme Scribe ou Bixio.

Le proviseur de Louis-le-Grand est confronté aux mêmes pressions et, passé le moment de la sanction, doit donner son accord, sous la pression des autorités, à la réintégration de nombre d'exclus dans d'autres établissements de la capitale : comme le dit le correspondant parisien d'un élève provincial, grâce à l'indulgence, " on peut tout espérer de la conduite future d'un adolescent bien né "59. On ne semble guère aimer, chez une bourgeoisie parisienne plutôt voltairienne, une éducation trop autoritaire et trop religieuse, et on le fait savoir bruyamment, sur le thème de l'erreur judiciaire. Mais s'exprime aussi tout l'enjeu de l'avenir professionnel de ces collégiens et de la quête d'un diplôme devenu nécessaire pour prendre la succession d'un père ou d'un oncle dans nombre de professions libérales. Dans les trois cas, la sanction finalement adoptée révèle des interférences

58 AN, $\mathrm{F}^{17}$ 8845, affaire Sainte-Barbe, rapport au conseil royal de l'Instruction publique, s.d. 
avec les réseaux relationnels traversant les milieux élitaires. À Amiens, le jeune du Sommerard, déjà rencontré, est transféré dans un autre collège, façon élégante d'éviter l'exclusion pure et simple, au motif " qu'une exclusion absolue au moment décisif de ses études le frapperait dans tout son avenir ; que la punition déjà subie doit avoir produit une impression salutaire sur un jeune cœur dont les penchants mal réprimés n'ont cependant pas étouffé les sentiments naturels vers le bien ${ }^{60}$; que d'ailleurs la position sociale d'une famille qui a donné d'honorables gages d'un dévouement sans réserve à la cause royale dans les moments les plus difficiles, mérite de justes égards " ${ }^{61}$.

De fait, si les renvois sont massifs (117 à Louis-le-Grand, plus de 130 à SainteBarbe), peu d'exclus restent en définitive sans affectation. À Sainte-Barbe, chaque élève doit produire une lettre faisant état de son repentir, demandant son pardon et s'engageant à ne pas récidiver. L'analyse du profil des exclus de Louis-le-Grand permet de dresser le portrait du collégien révolté moyen : pensionnaire, élève de quatrième, troisième ou seconde, âgé de 15 à 17 ans ${ }^{62}$. La ventilation des âges confirme que $30 \%$ sont âgés de 15 ans, $21 \%$ de 17 ans, $18 \%$ de 16 ans. Les plus jeunes comme les plus âgés sont faiblement représentés. Si les qualificatifs relatifs au caractère dominent (tête ardente, incorrigible, dissipé, fourbe, indiscipliné, etc.), ceux qui sont relatifs à la morale figurent en deuxième position : immoral, mœurs suspectes ou plus que suspectes, libertin, sans mœurs, de mauvaises mœurs, etc. Il devient alors difficile de diffuser ces listes : lorsqu'elles sont connues, elles génèrent de vigoureuses protestations de familles qui jugent infamants les jugements portés sur leur progéniture. Dans le cas d'Amiens, où le proviseur, l'abbé Ozouf, dresse une liste de dix-neuf collégiens à exclure, les annotations sont plus variées encore, touchant à la fois au social ("jeune homme riche ", "B.A. [boursier académique] à $3 / 4$ "), au familial (" sans père "), au scolaire (" paresse extraordinaire ", " paresse excessive "), au disciplinaire (" toujours cabalant "), au religieux (" a crié à ses camarades de ne pas répondre à la prière "), morale (" coupable d’une grande faute contre les mœurs ", "surpris corrompant les petits ") ${ }^{63}$. La juxtaposition d'annotations négatives atteint son paroxysme dans le cas d'un élève de

60 Rappel discret du fait que le collégien a déjà été chassé de Saint-Louis pour des " mauvaises mœurs ".

$61 \mathrm{AN}, \mathrm{F}^{17}$ 7287, affaire du collège royal d'Amiens, Rapport préliminaire sur les faits généraux, 30 mars 1828.

62 AN, $\mathrm{F}^{17} 7287$, collège royal de Louis-le-Grand. Élèves exclus. Élèves pensionnaires exclus du collège royal Louis-le-Grand, s.d.

$63 \mathrm{AN}, \mathrm{F}^{17} 7287$, Rapport sur la situation du collège royal d'Amiens aux derniers jours de février 1828 , 3 mars 1828 . 
rhétorique ainsi signalé : "Yungmann aîné, élève de rhétorique, B.A. à pens. ent. [pension entière], toujours en insurrection, mœurs douteuses, ayant de mauvais livres, les ouvrages les plus obscènes ; prenant les assiettes de la maison pour les fondre, faisant des balles, ayant un pistolet, de la poudre, favorisé pour cela par son titre de sacristain. Tout fut découvert, il eut l'impudence de tout réclamer à Mr l'Aumônier "64.

La mention de la possession d'armes à feu est rare : on rapprochera cette affaire de celle de Sainte-Barbe, où l'on apprend incidemment que les élèves de classe préparatoire étaient armés de pierres et de quatre fusils, dont ils ne firent pas usage, ceux-ci n'étant vraisemblablement pas chargés, mais sur la provenance desquels on ne s'interroge pas ${ }^{65}$. Dans le cas d'Amiens, on reste évidemment surpris d'un portrait qui fait d'un collégien un carbonaro en puissance, ce qui, par contrecoup, justifie les mesures d'exclusion proposées par le proviseur, mais en donnant du collège l'image d'un repaire de révolutionnaires aux mœurs douteuses. Concernant Yungmann, il s'avère que les lectures obscènes qui lui sont reprochées sont les Contes de La Fontaine et qu'il aurait été surpris dans la sacristie "dans le désordre le plus honteux " avec un autre élève ou, pour reprendre l'expression du proviseur, "dans l'acte même de la corruption avec un de ses camarades " ${ }^{66}$. La qualité de clerc du proviseur - alors que le censeur, dont on fustige l'absence d'autorité, est un laïc - est à prendre en considération : en 1828, au lendemain de la défaite électorale de Villèle et de la nomination de Martignac à la tête du gouvernement, un mouvement anticlérical assez fort se dessine dans l'Université en général, et chez les jeunes élites en particulier. L'affaire a aussi un aspect politique, dans la mesure où elle met en branle les autorités et les notabilités locales, qui s'offusquent de l'image donnée du collège de leur ville, et par conséquence des mœurs de leurs enfants. L'image même du proviseur pâtit lorsqu'on apprend qu'il a, de sa propre initiative, brûlé de mauvais livres trouvés dans un pupitre, que l'infirmerie, laissée sans surveillance, constituait le lieu central de corruption entre les élèves et que la sacristie était tout à la fois " le dépôt des livres obscènes du collège et le lieu d'asile des élèves corrompus " ${ }^{67}$. Aspect politique, toujours, lorsqu'il apparaît que le dénonciateur du prétendu complot, dont l'existence semble

64 Id. La fonction de sacristain est réservée à des élèves témoignant d'une bonne conduite et d'une certaine disposition à la piété.

65 AN, $F^{17} 8845$, affaire de Sainte-Barbe, rapport pour le Conseil royal, s.d.

$66 \mathrm{AN}, \mathrm{F}^{17} 7287$, lettre du proviseur au ministre de l'Instruction publique, 9 mars 1828.

67 AN, $\mathrm{F}^{17} 7287$, affaire du collège royal d'Amiens, Rapport préliminaire sur les faits généraux, 30 mars 1828. 
finalement douteuse, appartient à une famille " dont les opinions libérales sont fort prononcées " ${ }^{68}$.

À travers ces quelques exemples, se dessine donc une évolution récurrente du mode de gestion de la révolte collégienne : au plan discursif, l'affirmation d'une volonté répressive sans faille est affirmée dans un premier temps, avant que, sous la pression conjuguée des familles et de diverses notabilités appartenant aux mêmes catégories sociales, l'indulgence revienne au premier plan ; au plan pratique, ces interférences multiples viennent réduire à peu de chose, malgré des exceptions, les sanctions prises contre les rebelles. On s'éloigne donc rapidement de la régulation des émeutes ouvrières qui, si elle n'ignore pas non plus la modulation des sanctions en fonction de paramètres multiples (la prise en compte du repentir en fait partie), se montre beaucoup plus sévère, y compris dans des cas où les dégradations matérielles apparaissent bénignes. Cela revient, en conclusion, à poser de nouveau la question de la politisation de ces révoltes collégiennes : rarement reconnues comme politiques, elles sont le plus souvent ramenées à une simple éruption de violence juvénile, dont les vrais coupables sont identifiés comme des éléments extérieurs cherchant à corrompre des esprits prompts à s'enflammer. Éléments extérieurs qui, du reste, ne sont pas nommés, ni comme individus, ni comme catégorie : en cas de rupture de l'ordre légal dans le cadre d'une institution aussi surveillée que l'école, le principe d'extériorisation de la responsabilité appartient au régime classique de la rhétorique administrative et/ou politique.

Le collège ou le lycée représente toutefois comme une première expérience de mobilisation ou d'engagement politique pour de futures élites politiques : c'est ainsi que Godefroy Cavaignac, alors qu'il était pensionnaire à Sainte-Barbe en 1815, prend la tête des partisans de Napoléon, les bleus, qui affrontent violemment ceux de Louis XVIII, les blancs ${ }^{69}$. Mais il s'agit plutôt d'une exception, comme c'est aussi le cas sous la Restauration ou sous la monarchie de Juillet. La plupart de ces révoltes nous renseignent tout autant, sinon davantage, sur la confrontation à la fois générationnelle, sociale et culturelle, qui oppose deux formes de sensibilité à l'autorité, à la discipline, à l'obéissance, avec une indéniable connotation

$68 I d$.

69 Werdet, Souvenirs de Sainte-Barbe, cité in Godefroy Cavaignac, Romans militaires, Paris, C. Vanier, libraire-éditeur, $5^{\mathrm{e}}$ édition, 1868, pp. VIII-XVI. 
anticléricale. Le collège ou le lycée apparaît bien comme le lieu où se dessine un idéal libéral porté par des élites juvéniles, dans un esprit d'individualisme difficile à réduire à une uniformisation des pratiques et des contraintes de tous ordres. On ne peut toutefois s'empêcher de penser que, parvenus à des postes de responsabilité, y compris politique ou administrative, ces élites sociales n'agirent guère dans le sens d'un assouplissement des règlements en vigueur dans ces établissements. Comme si, en définitive, à côté d'une mission de formation, la mission disciplinaire de l'enseignement secondaire demeurait une constante que personne ne se risquait à remettre en cause, au-delà d'aménagements comme ceux qui seront instaurés par la loi de 1854 .

Jean-Claude CARON

Université Blaise-Pascal-Clermont-Ferrand II jeanclaudecaron@free.fr 